\title{
IMPACT OF POLARIMETRIC SAR SPECKLE REDUCTION ON CLASSIFICATION OF AGRICULTURE LANDS
}

\author{
R. Farhadiani ${ }^{1, *}$, S. Homayouni ${ }^{2}$, A. Safari ${ }^{3}$ \\ ${ }^{1,3}$ School of Surveying and Geospatial Engineering, College of Engineering, University of Tehran, Tehran, Iran - (r.farhadiani, \\ asafari)@ut.ac.ir \\ ${ }^{2}$ Centre Eau Terre Environnement, Institut National de la Recherche Scientifique, Quebec, Canada - saeid.homayouni@ete.inrs.ca
}

Commission VI, WG VI/4

KEY WORDS: Synthetic Aperture Radar, De-speckling, Classification, Support Vector Machine, Crop Map

\begin{abstract}
:
Presence of speckle in the Polarimetric Synthetic Aperture Radar (PolSAR) images could decrease the performance of information extraction applications such as classification, segmentation, change detection, etc. Hence, an essential pre-processing step named despeckling is needed to suppress this granular noise-like phenomenon from the PolSAR images. In this paper, a comparison study is conducted between several new PolSAR speckle reduction methods such as POSSC, PNGF, and ANLM. For this comparison, a 4look L-band AIRSAR NASA/JPL PolSAR dataset that obtained over an agriculture land from Flevoland, Netherlands, was employed. The de-speckling assessment was completed based on some no-reference quantitative indicators. All the de-speckling methods were evaluated in terms of speckle reduction form homogeneous areas, details, and radiometric preservation, and retaining the polarimetric information. Furthermore, the impact of PolSAR de-speckling on classification was evaluated. For this purpose, Support Vector Machine (SVM) classifier was used to classify H/A/Alpha decomposition. Experimental results showed that the ANLM method was better to suppress the speckle, followed by the PNGF method. Also, the classification results showed that a proper PolSAR de-speckling could effectively increase the classification accuracy. The improvement of the Overall Accuracy based on de-speckling using the ANLM method was approximately $22 \%$ and $13 \%$ higher than the POSSC and PNGF methods, respectively.
\end{abstract}

\section{INTRODUCTION}

The Polarimetric Synthetic Aperture Radar (PolSAR) systems are an active microwave imaging technique. The remotely sensed data which is collected by these systems is one of the most critical information resources that can gather crucial information from the earth surface. These systems can emit the fully polarized long-wavelength radar waves and receive the returned waves from the targets. As a result, all-weather, day and night imaging are the advantages of these systems. Consequently, the PolSAR data has been becoming an ideal tool for remote sensing applications. However, besides these superiorities over the optical imaging systems, SAR images are affected by a granular noise-like phenomenon named speckle, which is originated by the coherent nature of the SAR systems. Presence of the speckle in the PolSAR images degrades their radiometric quality (Lee, 1981). Moreover, speckle complicates image analysis and interpretation (Lee et al., 1999). Furthermore, the accuracy of the end-user products is highly dependent on the quality and reliability of the input data (Lopes et al., 1993) For this reason, an essential pre-processing step called de-speckling is needed to suppress the speckle from the PolSAR data.

In order to make a worthy trade-off between speckle reduction and details preservation, various methods have been proposed in the literature to reduce the speckle from the SAR images in general (Farhadiani et al., 2019; Gleich, 2018; Liu et al., 2018), and PolSAR images in particular (Lee et al., 2015; Ma et al., 2018; Nie et al., 2016). Thanks to the Non-Local Means (NLM) denoising approach (Buades et al., 2005) and its development for de-speckling the PolSAR data, many various non-local PolSAR speckle reduction methods have been proposed in the literature
(Ma et al., 2019; Shen et al., 2018; Xing et al., 2017; Xu et al., 2016; Yan et al., 2017).

Until now, several papers have been presented in the literature about PolSAR despeckling. However, only a few of them used a real-world application to show how de-speckling could affect the performance of the remote sensing applications (Lavreniuk et al., 2017; Lee et al., 1999; Mahdianpari et al., 2017; Medasani and Reddy, 2018). The main goal of this paper is to compare and assess the performance of several new PolSAR de-speckling methods, such as POSSC (Xu et al., 2016), PNGF (Ma et al., 2018) and ANLM (Shen et al., 2018) from different aspects. Beside this comparison, due to the importance of crop classification and achieving reliable crop map, we will also assess the influence of PolSAR de-speckling on the classification accuracy. To this end, Support Vector Machine (SVM) classifier (Cortes and Vapnik, 1995) has been employed to classify H/A/Alpha decomposition (Cloude and Pottier, 1997). Figure 1 shows the workflow of the proposed methodology.

\section{STATISTICS OF POLSAR DATA}

In a PolSAR data, the polarimetric information of the target is represented by a complex matrix named Sinclair scattering matrix as:

$$
S=\left[\begin{array}{ll}
S_{h h} & S_{h v} \\
S_{v h} & S_{v v}
\end{array}\right]
$$

\footnotetext{
* Corresponding author
} 


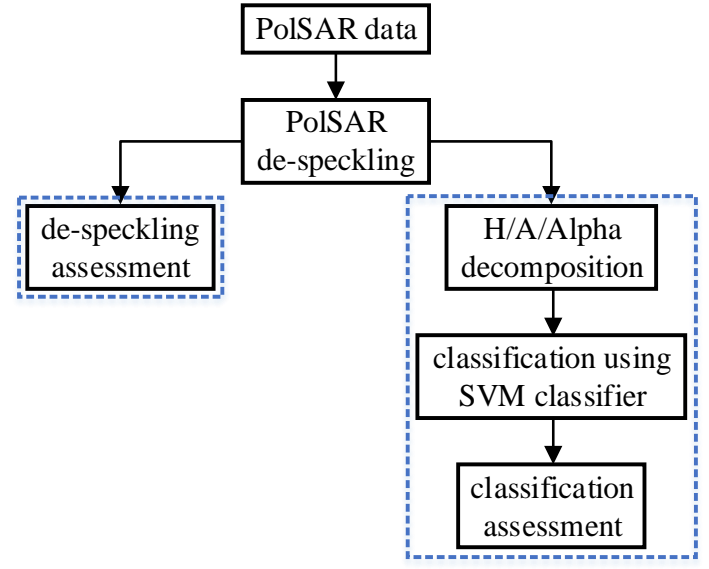

Figure 1. The workflow of this paper.

where $S_{k l}$ represents the scattering element of $k$ transmitting and $l$ receiving polarizations. In the case of reciprocal backscattering, we have $S_{h v}=S_{v h}$. Also, the polarimetric information can be denoted by a complex vector called the target scattering vector, which can be expressed as:

$$
\Omega=\left[\begin{array}{lll}
S_{h h} & \sqrt{2} S_{h v} & S_{v v}
\end{array}\right]^{T}
$$

where superscript $T$ stands for the matrix transpose (Lee and Pottier, 2009). Besides of Sinclair scattering matrix and target scattering vector, the polarimetric information can be indicated by a matrix named Covariance matrix $(\mathrm{C} 3)$ which is generated by the outer product of the target scattering vector with its conjugate transpose. The $\mathrm{C} 3$ matrix can be expressed as:

$$
\begin{aligned}
& \left\langle\mathrm{C}_{3}\right\rangle=\left\langle\Omega \cdot \Omega^{* T}\right\rangle \\
& =\left[\begin{array}{ccc}
\left\langle\left|S_{h h}\right|^{2}\right\rangle & \sqrt{2}\left\langle S_{h h} S_{h v}^{*}\right\rangle & \left\langle S_{h h} S_{v v}^{*}\right\rangle \\
\sqrt{2}\left\langle S_{h v} S_{h h}^{*}\right\rangle & 2\left\langle\left|S_{h v}\right|^{2}\right\rangle & \sqrt{2}\left\langle S_{h v} S_{v v}^{*}\right\rangle \\
\left\langle S_{v v} S_{h h}^{*}\right\rangle & \sqrt{2}\left\langle S_{v v} S_{h v}^{*}\right\rangle & \left\langle\left|S_{v v}\right|^{2}\right\rangle
\end{array}\right]
\end{aligned}
$$

where the subscript $*$ denotes the complex conjugate of a complex quantity and <.> indicates spatial ensemble averaging (Lopez-Martinez and Fabregas, 2003). It is proven that the covariance matrix follows the complex Wishart distribution (Goodman, 1963):

$$
P_{\mathrm{C}}(\mathrm{C})=\frac{L^{q L}|\mathrm{C}|^{L-q} \exp \left(-L \operatorname{Tr}\left(\Sigma^{-1} \mathrm{C}\right)\right)}{K(L, q)|\Sigma|^{L}}
$$

where $L$ is the equivalent number of looks (ENL), $q$ is the dimension of the polarimetric Covariance matrix, $|$.$| is the$ determinant operator, $\operatorname{Tr}($.$) is the trace of the matrix, \Sigma$ is the expectation of $\mathrm{C}$, and $K$ is a normalization factor, and it is equal to:

$$
K(L, q)=\pi^{\frac{q(q-1)}{2}} \prod_{j=1}^{q} \Gamma(L-j+1)
$$

where $\Gamma($.$) is the Gamma function. In the case of PolSAR data,$ the ENL can be estimated as proposed by Anfinsen et al.
(Anfinsen et al., 2009). It should be noted that in the intensity or amplitude SAR images, speckle has a multiplicative nature (Bianchi et al., 2013). However, in PolSAR data, speckle appears in the intensity images of each polarization, as well as in the complex cross-product terms (Lopez-Martinez and Fabregas, 2003). For this reason, the speckle in the PolSAR data is more complicated.

\section{POLSAR DE-SPECKLING}

In this section, we will introduce some recent PolSAR despeckling approaches, named the POSSC, PNGF, and the ANLM. It should be noted here that we will not describe these methods in detail. However, in the following, a brief description of these methods is presented.

\subsection{POSSC}

$\mathrm{Xu}$ et al. developed a transform-domain speckle suppression method for PolSAR images based on patch ordering algorithm and simultaneous sparse coding (SSC). At first, a signaldependent additive noise model for PolSAR Covariance matrix is established. After that, based on the complex Wishart distribution, the noise variance for each element of the matrix is computed. At the next step, by extracting sliding patches and organizing them, the ordered patches are filtered by SSC. Finally, by reconstructing the filtered patches via reverse permutation and sub-image averaging, the final de-speckled image is achieved.

\subsection{PNGF}

Ma et al. proposed a guided filter with nonlinear weight kernels and adaptive filtering windows for speckle reduction from PolSAR images. The idea behind this method is summarized in two main steps. In the first step, a guidance image with a low level of speckle is generated by a local weighted average with employing the statistical trait of the speckled data. In the second step, the final de-speckled image is obtained by using the fully polarimetric traits based on the guidance image and the speckled image.

\subsection{ANLM}

Shen et al. developed an adaptive non-local means filter with the shape-adaptive (SA) patches matching and optimal similarity test (Simi-Test) threshold selection for reducing the speckle from PolSAR images. By combining the polarimetric likelihood ratio test for the Coherency matrices (PolLRT-CM) and the region growing (RG), the homogeneous and heterogeneous pixels in textured areas can be efficiently distinguished. Also, the adaptive threshold selection of Simi-Test is used to enhance the filtering performance both in the homogeneous and textured areas.

\subsection{Filter Parameter Tuning}

Tuning the parameters in each de-speckling method is one of the most challenging tasks which has a direct influence on the despeckling performance. In the POSSC method, there is only one unknown parameter that must be tuned, and it is the number of looks $(L)$. The other parameters are fixed throughout the algorithm. In this paper, the number of looks of the AIRSAR data is equal to 4 . In the PNGF method, there are two unknown parameters, one is $L$, and another is $t$ to specify the normalization parameters $t_{1}$ and $t_{2}$. Larger values of $t$ result in a more speckle reduction at the expense of worse levels of preserving details, and vice versa. Similar to the POSSC method, other existing parameters of the PNGF method are fixed in the algorithm. In this paper, $t$ is considered 0.6 to compensate between speckle 
reduction and retain details. For the ANLM method, several unknown parameters must be tuned like patch radius, search radius, etc. All the unknown parameters are tuned as the authors of the original paper have proposed. For more information about the unknown parameters of each mentioned de-speckling methods, we refer the readers to their original articles.

\section{EXPERIMENTAL RESULTS}

In this paper, a real PolSAR data is used for the comparison of de-speckling methods. This dataset is acquired by the 4-look Lband NASA/JPL AIRSAR system that is obtained over a large agriculture land from Flevoland, Netherlands ${ }^{1}$ and covers various types of crops, some man-made structures and a small area of water. Figure 2 presented the Pauli RGB image of this dataset. Due to inaccessibility to the speckle-free PolSAR data, we will use some no-reference indicators, e.g., Equivalent Number of Looks (ENL), Edge-Preservation Degree based on Ratio of Average (EPD-ROA), Mean of Ratio image (MoR) and complex correlation coefficient $(\rho)$ for the evaluation purposes. Also due to the importance of the crop classification, we will classify the PolSAR images via Support Vector Machine (SVM) and evaluate the results based on Overall Accuracy (OA), Kappa coefficient, Producer's and User's accuracies.

\subsection{PolSAR De-speckling Assessment}

Indeed, an excellent de-speckling approach should smooth the homogeneous areas, preserve the details, avoid radiometric distortion, and artifact-free (Oliver and Quegan, 2004). Besides these properties, an excellent PolSAR filtering method should also preserve the polarimetric information (Xing et al., 2017). In the rest of this subsection, we will describe how we can assess a de-speckling method from these aspects.

ENL is widely used to assessing the de-speckling methods in terms of speckle reduction in a homogeneous area and can be expressed as:

$$
\mathrm{ENL}=(\mu / \sigma)^{2}
$$

where $\mu$ and $\sigma$ are the mean and standard deviation which are calculated from a homogeneous area of the SAR image, respectively. The higher value of the ENL denotes more speckle reduction from the homogeneous areas. In this paper, we computed the ENL in two different homogeneous areas. Another metric which is employed to evaluate the capability of the methods in terms of edge-preserving is EPD-ROA, and it is equal to (Feng et al., 2011):

$$
\mathrm{EPD}-\mathrm{ROA}=\frac{\sum_{i=1}^{N}\left|I_{S 1}(i) / I_{S 2}(i)\right|}{\sum_{i=1}^{N}\left|I_{I 1}(i) / I_{I 2}(i)\right|}
$$

where $I_{S 1}$ and $I_{S 2}$ denote the adjacent pixel values of the despeckled image along the horizontal or vertical direction, whereas $I_{I 1}$ and $I_{I 2}$ represent the corresponding nearby pixel values of the SAR image, respectively. A better edge-preserving method should have EPD-ROA value close to 1 . To evaluate the performance of de-speckling methods in terms of radiometric preservation, we will use Mean of Ratio image (MoR) which is computed based on the mean of the point-wise division of the SAR image before and after the speckle reduction process. In the

\begin{tabular}{|c|c|c|c|c|c|}
\hline \multicolumn{2}{|c|}{ Methods } & \multirow{2}{*}{ 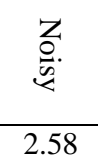 } & \multirow{2}{*}{$\begin{array}{l}0 \\
0 \\
\tilde{N} \\
\tilde{N} \\
13.08\end{array}$} & \multirow{2}{*}{$\begin{array}{c}\vec{\nabla} \\
\stackrel{2}{1} \\
17.34\end{array}$} & \multirow{2}{*}{$\begin{array}{l}\text { 麦 } \\
\text { 学 } \\
39.36 \\
\end{array}$} \\
\hline & Zone 1 & & & & \\
\hline ENL & Zone 2 & 2.76 & 16.78 & 22.80 & 56.36 \\
\hline \multirow{2}{*}{ EPD-ROA } & Hori. & - & 0.524 & 0.538 & 0.588 \\
\hline & Vert. & - & 0.475 & 0.481 & 0.505 \\
\hline \multicolumn{2}{|l|}{ MoR } & - & 0.962 & 0.982 & 0.983 \\
\hline \multicolumn{2}{|l|}{$|\rho(\mathrm{hh}, \mathrm{vv})|$} & 0.739 & 0.739 & 0.739 & 0.741 \\
\hline \multicolumn{2}{|l|}{$|\rho(\mathrm{hh}, \mathrm{hv})|$} & 0.074 & 0.075 & 0.073 & 0.073 \\
\hline \multicolumn{2}{|l|}{$|\rho(\mathrm{vv}, \mathrm{hv})|$} & 0.089 & 0.089 & 0.084 & 0.086 \\
\hline \multicolumn{2}{|l|}{$\angle \rho(\mathrm{hh}, \mathrm{vv})$} & -0.359 & -0.357 & -0.363 & -0.367 \\
\hline \multicolumn{2}{|l|}{$\angle \rho$ (hh,hv) } & 1.085 & 1.110 & 1.122 & 1.167 \\
\hline \multicolumn{2}{|l|}{$\angle \rho(\mathrm{vv}, \mathrm{hv})$} & 1.175 & 1.185 & 1.211 & 1.185 \\
\hline
\end{tabular}
ideal case, the MoR value should close to 1 .
Table 1. ENL, EPD-ROA, MoR, and $\rho$ values.

A suitable PolSAR de-speckling method should preserve the polarimetric information. For this reason, we will use the complex correlation coefficient to evaluate the polarimetric preservation capability of the de-speckling methods as follows (Lopez-Martinez and Fabregas, 2003):

$$
\rho_{i, j}=\frac{\left\langle S_{i} S_{j}^{*}\right\rangle}{\sqrt{\left\langle\left. S_{i}\right|^{2}\right\rangle\left\langle\left|S_{j}\right|^{2}\right\rangle}}
$$

where $i$ and $j$ represent two polarimetric channels. We computed this parameter in a homogeneous region. The amplitudes (|.|) and phase angles $(\angle)$ are estimated separately. In the ideal case, the complex correlation of the filtered image should be closed to the complex correlation of the original image that contains speckle. In this paper, $\rho$ is computed between hh and vv, hh and hv and vv and hv channels.

The Pauli RGB images of the speckled Flevoland data and despeckled images using the POSSC, PNGF, and the ANLM methods are presented in Figure 2. Also, Table 1 denotes the computed values of the previously mentioned de-speckling assessment indicators. It should be noted that we computed the ENL, EPD-ROA, and MoR indicators based on the diagonal elements of the $\mathrm{C} 3$ matrix and their averaged values were used for comparison. By investigation of the ENL values, we can find that the ANLM method could efficiently decrease the speckle from homogeneous areas. This can be seen in Figure 3 that represents the zoomed area of the noisy and de-speckled images. In the case of edge-preserving, the ANLM method was the best among other approaches, followed by PNGF method. However, some annoying granular-like artifact has been appeared in the PNGF de-speckling result, as can be seen in Figure 3. In the case of radiometric preservation, all methods had the acceptable ability. It should be noted that accurately tuning the parameters of a de-speckling method can dramatically increase the performance of a speckle reduction method. For example, if we increase the $t$ parameter in the PNGF method, the image will be more smoothed at the expense of losing details.

\subsection{Classification Assessment}

In this subsection, we will classify the PolSAR data to assess how de-speckling could affect the classification result. It should be pointed out here that the purpose of the classification in this paper is not to discuss which classifier is better or best.

\footnotetext{
${ }^{1}$ https://earth.esa.int/web/polsarpro/data-sources/sample datasets
} 

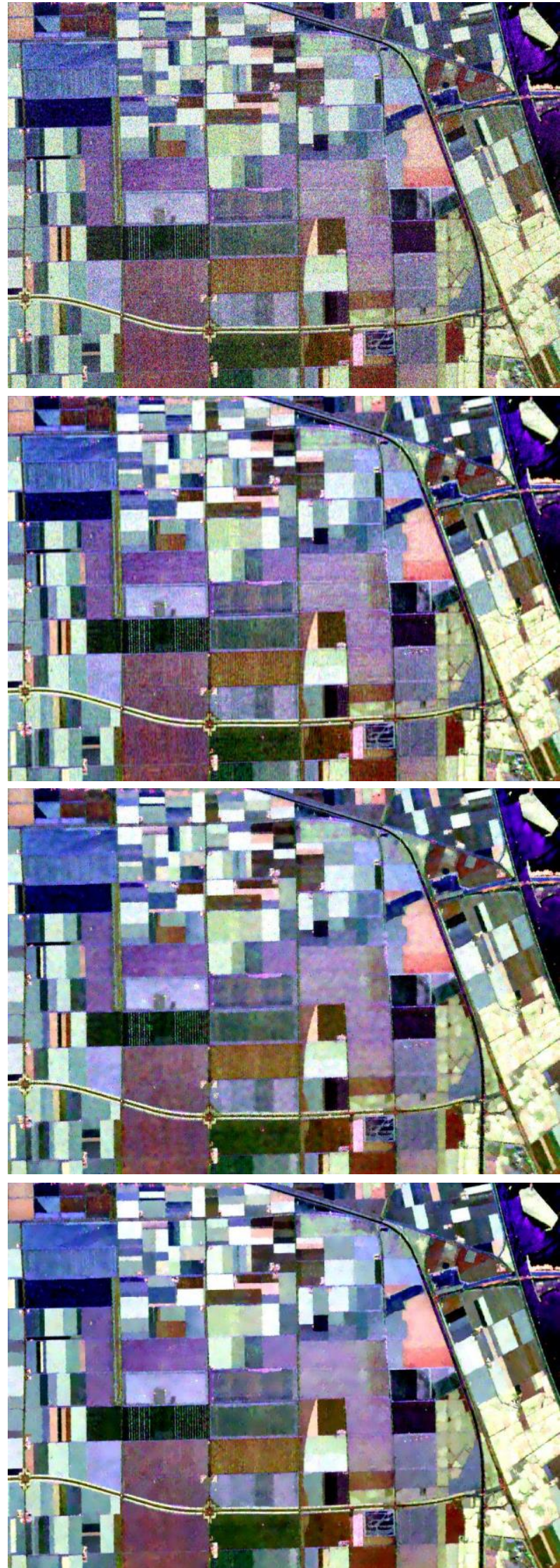

Figure 2. Pauli RGB images. From top to down, the speckled data, the POSSC de-speckled data, the PNGF de-speckled data, and the ANLM de-speckled data, respectively.

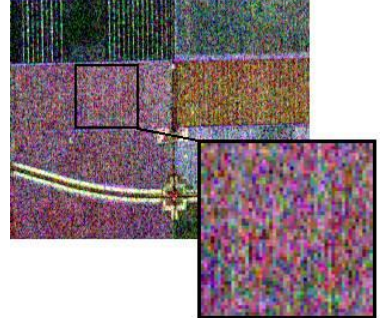

(a)

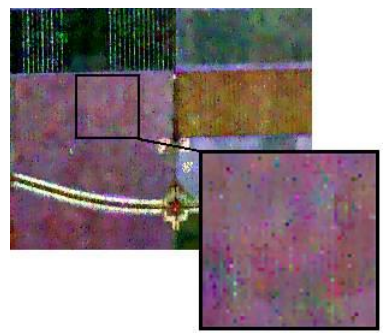

(c)

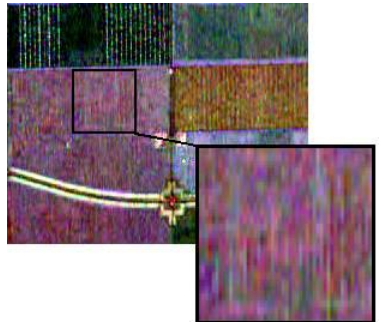

(b)

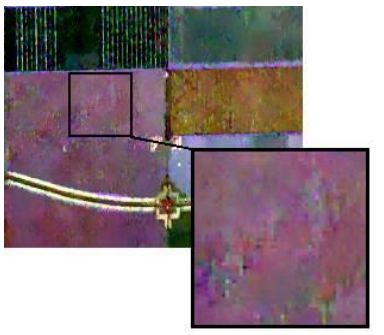

(d)
Figure 3. The zoomed area images. (a) speckled data, (b) POSSC, (c) PNGF, and (d) ANLM.

\begin{tabular}{|l|c|c|}
\cline { 2 - 3 } \multicolumn{1}{c|}{} & $\begin{array}{c}\text { \# of } \\
\text { training data }\end{array}$ & $\begin{array}{c}\text { \# of } \\
\text { test data }\end{array}$ \\
\hline Bare Soil & 1202 & 6011 \\
\hline Barely & 1583 & 7916 \\
\hline Beet & 2462 & 12310 \\
\hline Building & 134 & 673 \\
\hline Forest & 3370 & 16851 \\
\hline Grass & 1753 & 8766 \\
\hline Lucerne & 1989 & 9946 \\
\hline Peas & 2103 & 10519 \\
\hline Potatoes & 3752 & 18764 \\
\hline Rapeseed & 3885 & 19428 \\
\hline Stem Beans & 1667 & 8339 \\
\hline Water & 2067 & 10339 \\
\hline Wheat A & 3482 & 17410 \\
\hline Wheat B & 2390 & 11952 \\
\hline Wheat C & 4484 & 22421 \\
\hline
\end{tabular}

Table 2. The number of training and test data.

As a result, it does not matter that which classifier is employed for the classification, but the classification conditions (e.g. tuned parameters, number of training data, etc.) must be equal and constant for all PolSAR datasets which are de-speckled based on the POSSC, PNGF, and the ANLM methods. In this way, the comparison of the classification results will be fair.

In this paper, the Support Vector Machine (SVM) classifier is employed to classify the AIRSAR data. The description of the SVM algorithm is out of the scope of this paper, so we will refer the readers to the original article (Cortes and Vapnik, 1995) for more details about how SVM works. We employed LibSVM to classify the PolSAR data (Chang and Lin, 2011). The SVM uses the Radial Basis Function (RBF) kernel, also $C$ and $\gamma$ parameters have been fixed to 1000 and 0.125 , respectively. Furthermore, the classification is done based on the H/A/Alpha decomposition.

In the Flevoland agriculture land, various types of classes, e.g. Bare Soil, Barely, Beet, Building, Forest, Grass, Lucerne, Peas, Potatoes, Rapeseed, Stem Beans, Water, and Wheat are existed (Uhlmann and Kiranyaz, 2014). Figure 4 represents the ground truth for this data. It should be noted that $20 \%$ of the ground truth data has been used for training the SVM. Table 2 denotes the number of training and test data for each class. 


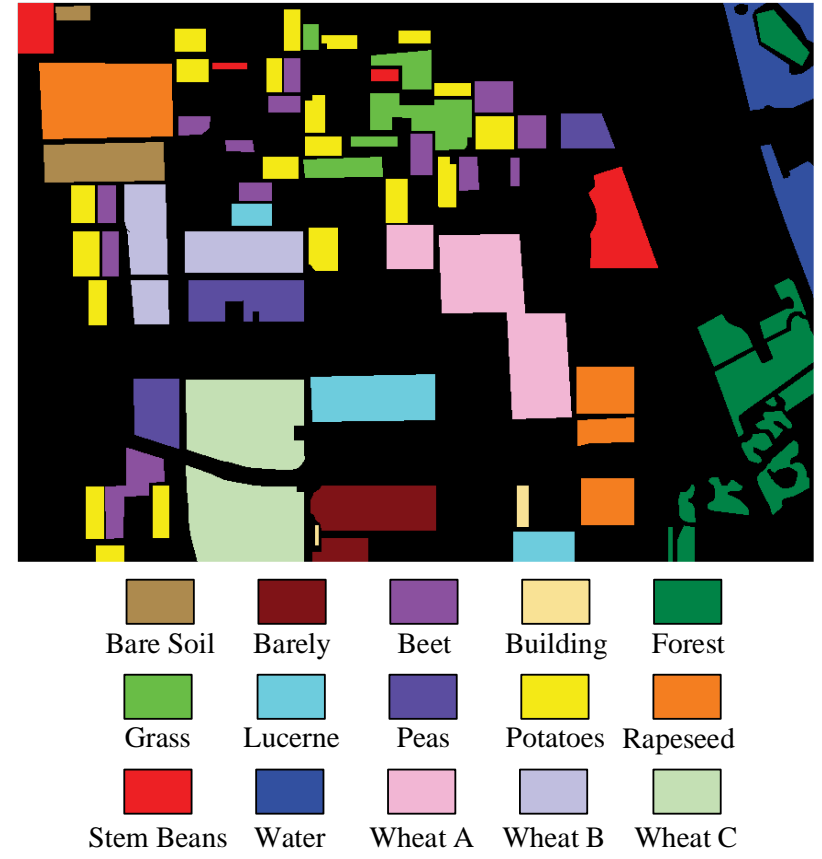

Figure 4. Ground truth for Flevoland area and its legend.

\begin{tabular}{|l|c|c|c|}
\cline { 2 - 4 } \multicolumn{1}{c|}{} & POSSC & PNGF & ANLM \\
\hline OA $(\%)$ & 53.77 & 62.30 & 75.40 \\
\hline Kappa & 0.49 & 0.59 & 0.73 \\
\hline
\end{tabular}

Table 3. Overall Accuracy and Kappa coefficient values based on different PolSAR de-speckling methods.

Overall Accuracy (OA), Kappa coefficient, Producer's and User's accuracies are employed for the classification assessment. All these metrics are computed based on the confusion matrix. OA is computed by dividing the diagonal elements of the confusion matrix (i.e. the total number of correctly classified pixels) to the total number of pixels (Foody, 2002). Also, the Kappa coefficient (Cohen, 1960) that uses all elements of the confusion matrix is employed for comparison. The higher OA and Kappa coefficient represents a better result in classification. The Producer's accuracy indicates how well a given class has been classified (Banko, 1998). Also, the User's accuracy denotes the reliability of the map and tell us how well the map represents what is really on the ground (Story and Congalton, 1986).

Figure 5 represents the final crop maps. Also, Figure 6 and Figure 7 illustrate the bar graphs of the computed Producer's and User's accuracies based on de-speckling using the POSSC, PNGF and the ANLM methods. Furthermore, Table 3 denotes the OA and Kappa coefficient values. According to Figure 5, the salt and pepper effect of the speckle is completely obvious in all classification results, especially in the classification result based on de-speckling via the POSSC method. This is because the POSSC method could not effectively suppress the speckle from the PolSAR data compared to other de-speckling methods. The ANLM method could achieve the best OA, among other approaches. Improvement of OA based on de-speckling using the ANLM method than the POSSC and PNGF methods was approximately around to $22 \%$ and $13 \%$, respectively. The reasonably good User's and Producer's accuracies for each class denoted the better performance of the classification using the ANLM method. As a result, we can say that if a de-speckling method has better speckle suppression, it could dramatically enhance the classification result.
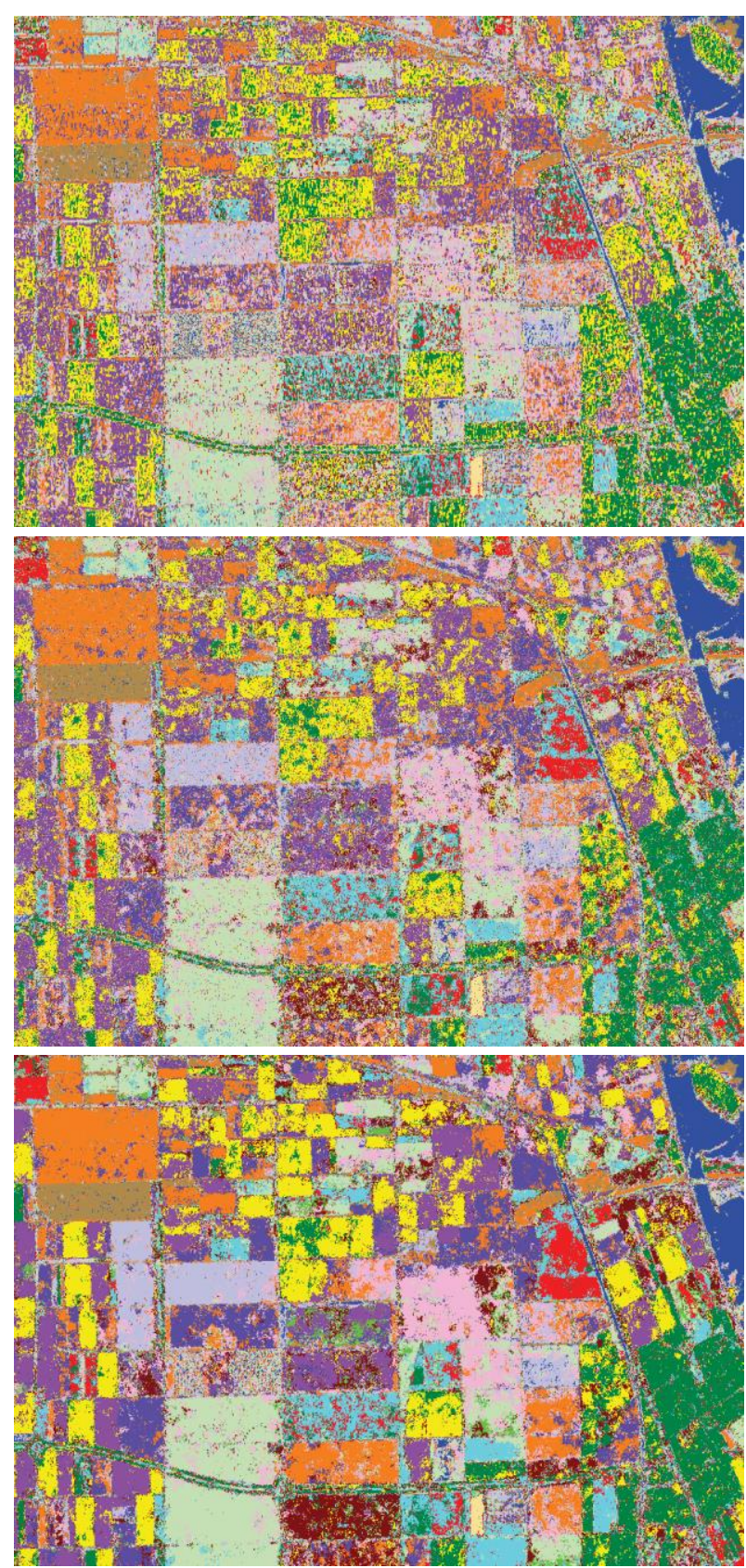

Figure 5. Classification results of the Flevoland area. From top to down, classification results based on the POSSC, PNGF, and

the ANLM PolSAR de-speckling methods, respectively.

However, besides the suitable speckle reduction method and tuning its parameters correctly, many other factors could affect the classification results, such as the accuracy and the number of the ground truth, tuning the classifier's parameters properly, extracting various features from PolSAR data and using them appropriately in the classification process, etc.

\section{CONCLUSION}

In this paper, a comparison study between some new PolSAR despeckling methods named the POSSC, PNGF, and the ANLM was done from two aspects, one assessing using some nonreferenced indicators, and the second assessment was to evaluate the classification results by employing SVM classifier. 


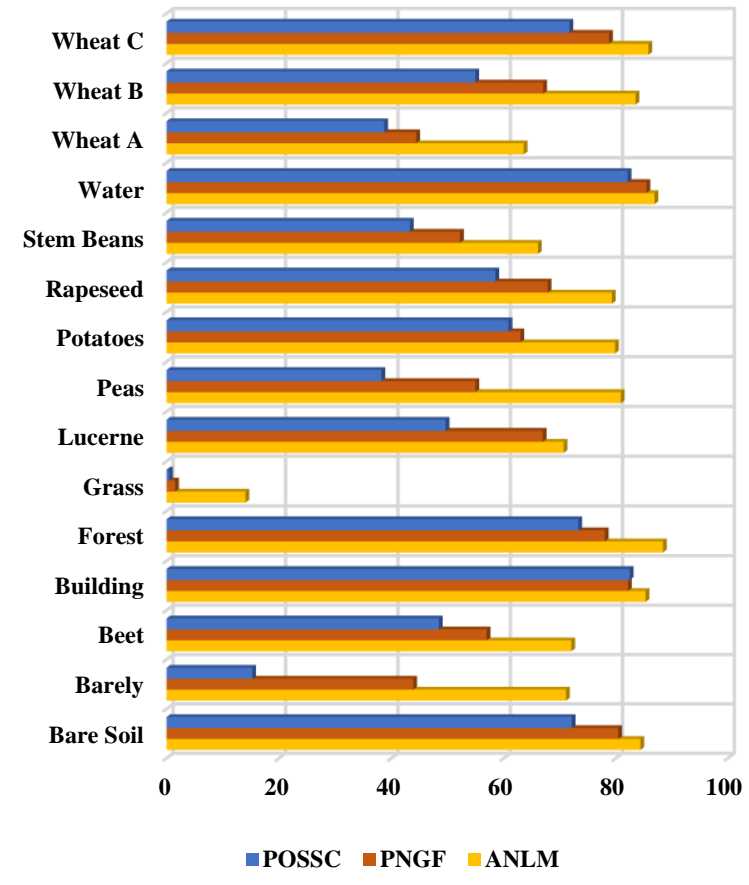

Figure 6. Comparison of Producer's accuracy based on various croplands obtained by different de-speckling methods.

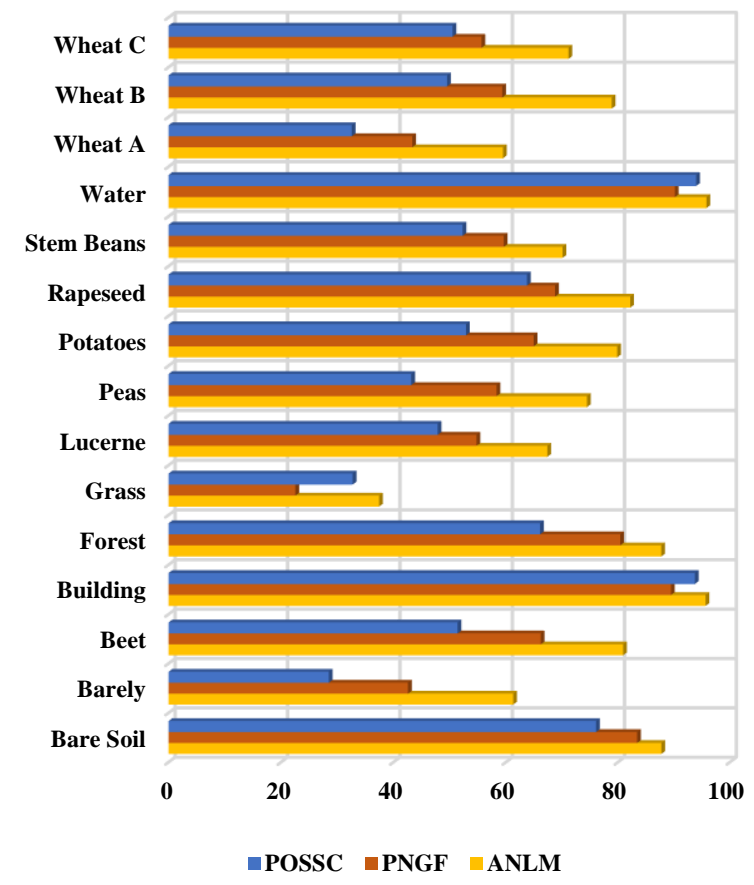

Figure 7. Comparison of User's accuracy based on various croplands obtained by different de-speckling methods.

Experimental results on the 4-look L-band AIRSAR data that obtained over an agriculture land revealed that the ANLM method has excellent ability to suppress the speckle from homogeneous areas, as well as preserve the details and polarimetric properties and avoid radiometric distortion. Also, the POSSC and PNGF methods had acceptable results in despeckling. Moreover, the classification result based on the ANLM method de-speckling had better OA than the other two methods. In the best case, the improvement in OA was approximately around $22 \%$.

\section{ACKNOWLEDGEMENTS}

The authors would like to thank the NASA/JPL for providing the AIRSAR data, as well as Chang et al. (2011) for providing the LibSVM library. Also, we would like to thank Xu et al. (2016), Ma et al. (2018) and Shen et al. (2018) for providing the codes of POSSC, PNGF, and ANLM methods, respectively.

\section{REFERENCES}

Anfinsen, S.N., Doulgeris, A.P., Eltoft, T., 2009. Estimation of the Equivalent Number of Looks in Polarimetric Synthetic Aperture Radar Imagery. IEEE Trans. Geosci. Remote Sens. $\quad 47$, 3795-3809. https://doi.org/10.1109/TGRS.2009.2019269

Banko, G., 1998. A review of assessing the accuracy of classifications of remotely sensed data and of methods including remote sensing data in forest inventory.

Bianchi, T., Argenti, F., Lapini, A., Alparone, L., 2013. Amplitude vs intensity despeckling in the wavelet domain using bayesian estimators, in: 2012 Tyrrhenian Workshop on Advances in Radar and Remote Sensing (TyWRRS). pp. 267-274. https://doi.org/10.1109/TyWRRS.2012.6381141

Buades, A., Coll, B., Morel, J.-., 2005. A non-local algorithm for image denoising, in: 2005 IEEE Computer Society Conference on Computer Vision and Pattern Recognition (CVPR'05). pp. 60-65 vol. 2 . https://doi.org/10.1109/CVPR.2005.38

Chang, C.-C., Lin, C.-J., 2011. LIBSVM: A library for support vector machines. ACM Trans. Intell. Syst. Technol. 2, 27.

Cloude, S.R., Pottier, E., 1997. An entropy based classification scheme for land applications of polarimetric SAR. IEEE Trans. Geosci. Remote Sens. 35, 68-78. https://doi.org/10.1109/36.551935

Cohen, J., 1960. A coefficient of agreement for nominal scales. Educ. Psychol. Meas. 20, 37-46.

Cortes, C., Vapnik, V., 1995. Support-Vector Networks. Mach. Learn. 20 ,

273-297. https://doi.org/10.1023/A:1022627411411

Farhadiani, R., Homayouni, S., Safari, A., 2019. Hybrid SAR Speckle Reduction Using Complex Wavelet Shrinkage and Non-Local PCA-Based Filtering. IEEE J. Sel. Top. Appl. Earth Obs. Remote Sens. 1-8. https://doi.org/10.1109/JSTARS.2019.2907655

Feng, H., Hou, B., Gong, M., 2011. SAR Image Despeckling Based on Local Homogeneous-Region Segmentation by Using Pixel-Relativity Measurement. IEEE Trans. Geosci. Remote Sens. 49, 2724-2737. https://doi.org/10.1109/TGRS.2011.2107915

Foody, G.M., 2002. Status of land cover classification accuracy assessment. Remote Sens. Environ. 80, 185-201. https://doi.org/https://doi.org/10.1016/S00344257(01)00295-4

Gleich, D., 2018. Optimal-Dual-Based\$1_1\$Analysis for Speckle Reduction of SAR Data. IEEE Trans. Geosci. Remote Sens. $\quad 56$, 6674-6685. https://doi.org/10.1109/TGRS.2018.2841191

Goodman, N.R., 1963. Statistical Analysis Based on a Certain Multivariate Complex Gaussian Distribution (An Introduction). Ann. Math. Stat. 34, 152-177. https://doi.org/10.1214/aoms/1177704250

Lavreniuk, M., Kussul, N., Meretsky, M., Lukin, V., Abramov, S., Rubel, O., 2017. Impact of SAR data filtering on crop 
classification accuracy, in: 2017 IEEE First Ukraine Conference on Electrical and Computer Engineering (UKRCON). pp. 912-917. https://doi.org/10.1109/UKRCON.2017.8100381

Lee, J.-S., 1981. Speckle analysis and smoothing of synthetic aperture radar images. Comput. Graph. Image Process. 17, 24-32. https://doi.org/https://doi.org/10.1016/S0146664X(81)80005-6

Lee, J.-S., Grunes, M.R., Grandi, G. de, 1999. Polarimetric SAR speckle filtering and its implication for classification. IEEE Trans. Geosci. Remote Sens. 37, 2363-2373. https://doi.org/10.1109/36.789635

Lee, J.-S., Pottier, E., 2009. Polarimetric radar imaging: from basics to applications. CRC press.

Lee, J., Ainsworth, T.L., Wang, Y., Chen, K., 2015. Polarimetric SAR Speckle Filtering and the Extended Sigma Filter. IEEE Trans. Geosci. Remote Sens. 53, 1150-1160. https://doi.org/10.1109/TGRS.2014.2335114

Liu, F., Wu, J., Li, L., Jiao, L., Hao, H., Zhang, X., 2018. A Hybrid Method of SAR Speckle Reduction Based on Geometric-Structural Block and Adaptive Neighborhood. IEEE Trans. Geosci. Remote Sens. 56, 730-748. https://doi.org/10.1109/TGRS.2017.2754420

Lopes, A., Nezry, E., Touzi, R., Laur, H., 1993. Structure detection and statistical adaptive speckle filtering in SAR images. Int. J. Remote Sens. 14, 1735-1758. https://doi.org/10.1080/01431169308953999

Lopez-Martinez, C., Fabregas, X., 2003. Polarimetric SAR speckle noise model. IEEE Trans. Geosci. Remote Sens. 41 , https://doi.org/10.1109/TGRS.2003.815240

Ma, X., Wu, P., Shen, H., 2019. Multifrequency Polarimetric SAR Image Despeckling by Iterative Nonlocal Means Based on a Space-Frequency Information Joint Covariance Matrix. IEEE J. Sel. Top. Appl. Earth Obs. Remote Sens. 12, 274-284. https://doi.org/10.1109/JSTARS.2018.2884194

Ma, X., Wu, P., Shen, H., 2018. A Nonlinear Guided Filter for Polarimetric SAR Image Despeckling. IEEE Trans. Geosci. Remote Sens. 57, 1918-1927. https://doi.org/10.1109/TGRS.2018.2870188

Mahdianpari, M., Salehi, B., Mohammadimanesh, F., 2017. The Effect of PolSAR Image De-speckling on Wetland Classification: Introducing a New Adaptive Method. Can. J. Remote Sens. 43, 485-503. https://doi.org/10.1080/07038992.2017.1381549

Medasani, S., Reddy, G.U., 2018. Speckle Filtering and its Influence on the Decomposition and Classification of Hybrid Polarimetric Data of RISAT-1. Remote Sens. Appl. Soc. Environ. 10, 1-6. https://doi.org/https://doi.org/10.1016/j.rsase.2018.02.002

Nie, X., Zhang, B., Chen, Y., Qiao, H., 2016. A New Algorithm for Optimizing TV-Based PolSAR Despeckling Model. IEEE Signal Process. Lett. 23, 1409-1413. https://doi.org/10.1109/LSP.2016.2602299

Oliver, C., Quegan, S., 2004. Understanding synthetic aperture radar images. SciTech Publishing.

Shen, P., Wang, C., Gao, H., Zhu, J., 2018. An Adaptive Nonlocal Mean Filter for PolSAR Data with ShapeAdaptive Patches Matching. Sensors (Basel). 18, 2215. https://doi.org/10.3390/s18072215

Story, M., Congalton, R.G., 1986. Accuracy assessment: a user's perspective. Photogramm. Eng. Remote Sensing 52, $397-$ 399.

Uhlmann, S., Kiranyaz, S., 2014. Integrating Color Features in Polarimetric SAR Image Classification. IEEE Trans. Geosci. Remote Sens. 52, 2197-2216.
https://doi.org/10.1109/TGRS.2013.2258675

Xing, X., Chen, Q., Yang, S., Liu, X., 2017. Feature-Based Nonlocal Polarimetric SAR Filtering. Remote Sens. . https://doi.org/10.3390/rs9101043

Xu, B., Cui, Y., Zuo, B., Yang, J., Song, J., 2016. Polarimetric SAR Image Filtering Based on Patch Ordering and Simultaneous Sparse Coding. IEEE Trans. Geosci. Remote Sens. 54, 4079-4093. https://doi.org/10.1109/TGRS.2016.2536648

Yan, T., Yang, W., Yang, X., López-Matínez, C., Li, H., Liao, M., 2017. Polarimetric SAR Despeckling by Integrating Stochastic Sampling and Contextual Patch Dissimilarity Exploration. IEEE J. Sel. Top. Appl. Earth Obs. Remote Sens. 10 , $2738-2753$ https://doi.org/10.1109/JSTARS.2017.2706500 